\title{
Molecular Biology Modernizing Planetary Protection Engineering to Enable Science for Biologically Sensitive Robotic and Human Missions
}

\author{
J. Nick Benardini \\ 818-354-4453 \\ Jet Propulsion Laboratory / California Institute of Technology (JPL/Caltech) \\ James.N.Benardini@jpl.nasa.gov \\ September 15, 2020
}

Co-authors/reviewers with respective institutions:

Nitin Singh (JPL/Caltech); Kasthuri Venkateswaran (JPL/Caltech)

Cosigners (14 at the time of submittal) can add their endorsements of this White Paper at:

https://docs.google.com/spreadsheets/d/10AKsx0naC2XYzD39CfWEHqFUW9EeOUddLS66UTeXg4k/edit \#gid=0

This work was carried out at the Jet Propulsion Laboratory, California Institute of Technology, under a contract with the National Aeronautics and Space Administration (80NM0018D0004). 


\section{Rationale}

Planetary Protection (PP) requirements have evolved and are currently evolving to meet the needs of increasingly sophisticated missions such as Human to Mars, Icy World exploration, and sample return from biologically sensitive target bodies. However, advances in biochemical, optical, and nucleic acid technologies have not yet found their way into standard PP practice and are being used only as research tools. Mars 2020 was the first mission to have a biological knowledge capture requirement that generated a "genetic inventory" or passenger list. For future missions, this new knowledge could be taken further, for example: to utilize the nucleic acid sequences to make implementation decisions in real-time, for semiquantitative analysis inputs, or for any high-resolution characterization of microbial traits and biochemical capabilities. Since the NASA Standard Assay (NSA), a culture-based technique, estimates only aerobic spore-formers and does not comprehensively address microbial diversity and the functional capabilities of microorganisms associated with the spacecraft surfaces, a broader technique is desired to understand the full breadth of viable organisms present on the spacecraft, including metabolic traits that could feed into probabilistic risk assessments. One way to have a broader microbial assessment is to measure and characterize biological contamination of surfaces by way of modern molecular techniques. A nucleic acidbased approach, metagenomics, would serve to fill this knowledge gap as it has been under development since the early 2000s in academia, as well as in medical, pharmaceutical, semiconductor, and aerospace industries, including NASA.

\section{Approach}

Since the 1960s Ranger Program, biologically sensitive missions required to adhere to PP policy have used the NSA method as the gold standard for assessing spacecraft biological cleanliness (1-4). The NSA method still continues to serve as the only approved technique to assess spacecraft biological cleanliness as spores have proven to be the most difficult to inactivate through environmental perturbations (e.g., ultraviolet [UV], space vacuum, radiation) $(5,6)$ and have demonstrated varying tolerance to spacecraft microbial reduction modalities (e.g., solvent cleaning and heat) (7). Additionally, the resulting NSA heat tolerant spore, acts as a hardy biological indicator unsurpassed in sensitivity, to the standard dry heat microbial reduction techniques for spacecraft. As a hardy biological indicator, the temperatures and times for the treatment effectively reduce all the viable non-spore population while reducing a portion or all of the spores. With the increase of sensitive optical, electronics, and commercial off-the-shelf components more and more components cannot undergo the standard treatment resulting in a broader non-spore viable population that may need to be characterized for more sensitive missions.

Techniques like 16S rRNA gene cloning (1980s), microarray (early 2000s), and targeted amplicon sequencing (2010s) were tried and utilized to measure the widest possible spectrum of microbes associated with spacecraft components (8), but these molecular methods are unable to characterize functional pathways that are essential for identifying PP-relevant microorganisms. PP relevant microorganisms by definition are specific for a given mission (9)to include resistance to: solvent cleaning, microbial reduction (e.g. standard dry heat processing), low nutrient cleanroom survivability, deep space conditions (i.e. thermal and vacuum resulting in extreme desiccation conditions, radiation), and operational environmental parameters (i.e. UV for Mars or ionizing radiation for Jovian missions). Microbial populations in hardware environments have been studied using environmental genomic inventory-based assessments, published in peer-reviewed journals (10-14). The published studies to date have noted six high-level observations:

1. A common set of microorganisms exists with about a $\sim 75 \%$ diversity overlap from facility to facility.

2. Geographically different cleanrooms (e.g., California vs. Florida) have distinct resident populations that reside outside the common core set of microorganisms. 
3. Populations are dynamic and tend to be ever-changing due to human and hardware activity.

4. Unique microbial profiles exist among potentially viable organisms and the total microbial diversity in the samples analyzed.

5. Molecular microbial diversity (sequences) generated from NASA cleanrooms constituted $\sim 20 \%$ of new microbial lineages that were not detected anywhere in the world before.

6. More than 50 novel microorganisms isolated from spacecraft hardware have been identified and described through whole genome sequencing methods.

Given these peer-reviewed journal observations, an increased microbial diversity assessment was recognized as a knowledge gap that needed be conducted broadly to understand microbial populations in a wide variety of cleanroom processing facilities. This is key to further expanding the knowledge base for the core microbiome and distinct resident microbial populations in a variety of facilities. Furthermore, this can provide PP engineers on biologically sensitive missions with an enhanced data set to make engineering judgments for development of the PP plan, tailored hardware-specific implementation, and verification approaches. Metagenomics could serve as another implementation approach to meeting PP requirements, without always utilizing the worst-case scenario approach, to enhance characterization of the actual microbial populations on the spacecraft hardware determined on a resolution basis of specific pieces of hardware. Technology advancements in microbiology and molecular biology in the past decade (Fig. 1) could be utilized to provide an enhanced genetic resolution on what specific biological contamination is present on the spacecraft and semi-quantitatively determine how much is present, which benefits all future missions by appreciably increasing spacecraft functionality, reliability, and mission success.

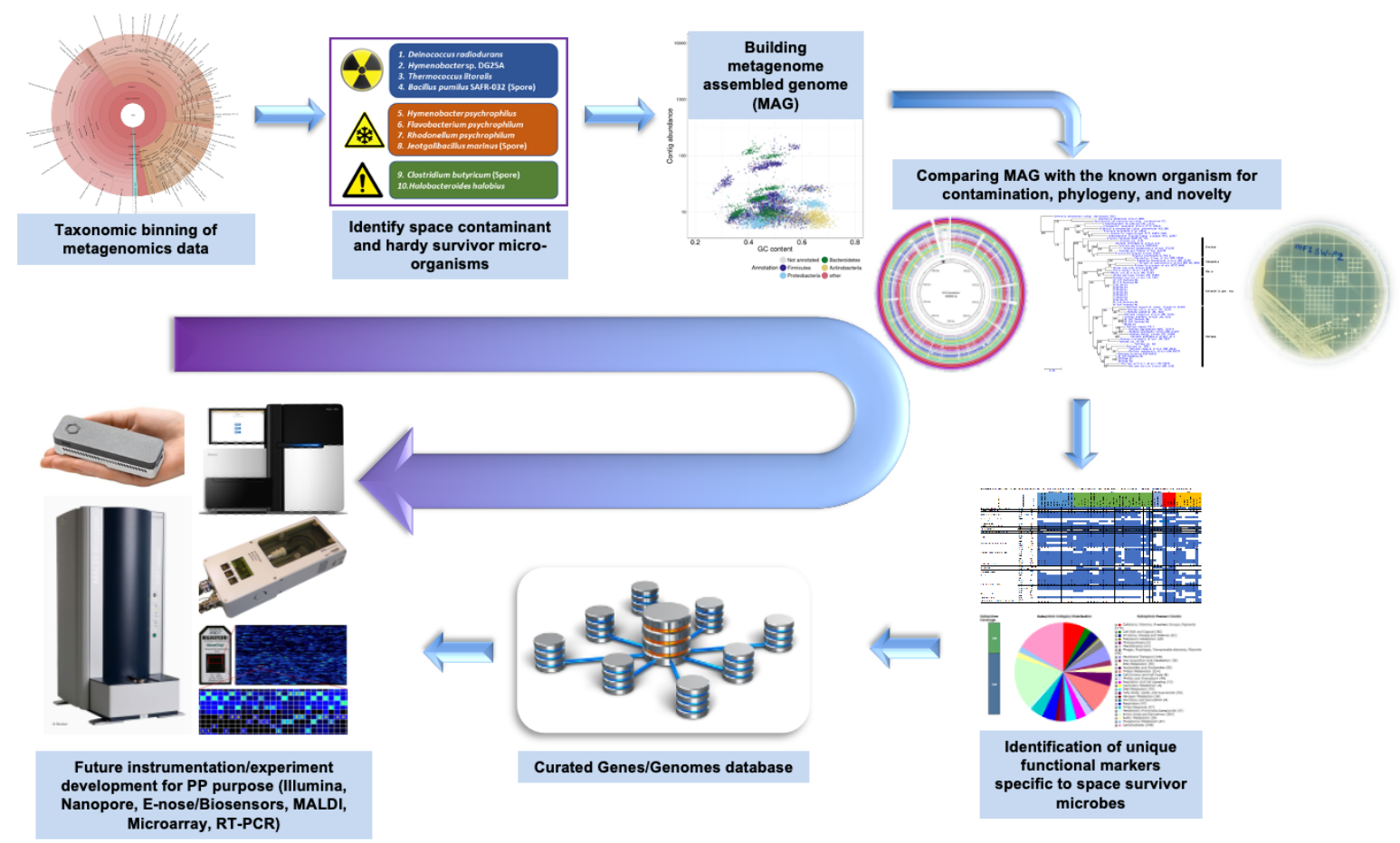

Figure 1. An example of the application of a modern-day nucleic acid-based approach for spacecraft Planetary Protection implementation. 


\section{Planetary Protection Knowledge Gaps}

\subsection{Culture-Based Biological Assessments}

\subsubsection{Current NASA Verification Approach}

The current culture-based NSA method is designed to assess a count of microorganisms that can form endospores, respire aerobically, are resistant to heat shock $\left(80^{\circ} \mathrm{C} ; 15 \mathrm{~min}\right)$, and can be cultivated in 72 hours on tryptic soy agar (TSA) media (9). While the total adenosine tri-phosphate (ATP) and limulus amebocyte lysate ( $L A L)$ assays are approved for pre-screening hardware for the presence of microbial contamination prior to conducting the NSA, these are the only two end-to-end industry techniques that NASA has investigated that have been incorporated into the PP handbook. It has been well established that organisms that are cultured by the NSA only account for $<0.1 \%$ of the overall microbial population present on a given spacecraft surface. From a biological perspective, we know that these hardy organisms represent an arguably small fraction of the total observed population on the spacecraft surfaces. Having the ability to differentiate the types of microbial population would greatly improve our ability to ensure that hardware undergoes the necessary microbial reduction, but not apply an overkill or a one-size-fits-all approach. Mathematically, these hardy or resistant organisms would be a driving parameter in the probability models and thus be the focus of subsequent engineering trades that are necessary to close the probabilistic risk-based requirements.

\subsubsection{Tailored Approach to Supplement Molecular Data}

A hybrid approach that combines both culture-based and modern nucleic acid-based methods allows for a deeper understanding of microbial tolerances to spacecraft sterilization or environmental conditions. A classic example of this for PP is using the Viking isolated Bacillus sp. ATCC 29669 to redefine the standards for high-heat processing (7). Metagenome-assembled genomes (MAG) can be used to identify uncultivable species. A previously published report shows that contemporary workers were able to identify the uncultivable organism Candidatus Amarolinea aalborgensis gen. nov., sp. nov., associated with a wastewater treatment plant via fluorescent in situ hybridization microscopy, 16S rRNA microbiome, and shotgun metagenomic sequence analysis, but they could not cultivate them to validly describe the microbial species (15). Another study, based on 16S rRNA microbiome analysis from a marine saltern pond in southwest Spain, revealed the presence of a dominant halophilic bacterium, enabling the researchers to define a suitable culture medium for successfully isolating and describing the novel genus Spiribacter salinus (16). A recent metagenomic study conducted on the International Space Station (ISS) provided near complete draft assemblages of seven genomes. Subsequently, based on the average nucleotide identity (ANI; $<95 \%$ ) and digital DNA-DNA hybridization ( $\mathrm{dDDH} ;<70 \%$ ) characterizations (17), four of these seven MAGs were phylogenetically affiliated to a novel genus. Furthermore, seven strains belonging to this novel genus were retrieved from the ISS culture collection, which were previously isolated from various locations of the ISS and archived (18). These archived isolates were further studied for phylogenetic similarity and biochemical traits directly illustrating the importance of the hybrid approach that uses both culture- and modern nucleic acid-based methods.

\subsection{Enumeration of Viable Biological Contamination}

Current shotgun metagenomic methods only provide a semi-quantitative assessment of the microbial populations, which is insufficient for establishing a microbial population distribution to verify PP requirements for the quantity of biological contamination or probabilistic risk models. As a knowledge gap, the current industrial and academic nucleic acid enumeration polymerase chain reaction (PCR) trade space needs to be evaluated to discern the most suitable approach to accomplishing this need for PP. A method that shows recent promise includes some combination of propidium monoazide to fractionate the viable spacecraft population, quantitative PCR (qPCR) to detect down to $\sim 10$ cells of a specific target based on 
generated primer (19), or a digital droplet PCR (ddPCR), which has shown increased precision and accuracy over qPCR (20).

\subsection{Phylogenetic Identification and High-Resolution Characterization of Microbial Traits and Biochemical Capabilities of Microorganisms on Spacecraft}

3.3.1 Establishing a Low-Biomass Standard Operating Protocol

In early 2000s, the genetic target for compiling the inventory was the hypervariable regions of the 16S rDNA gene that, for bacterial and archaeal sequences, provides the ability to discriminate among organisms at the operational taxonomic unit (OTU) level-an approach originally proposed by Pace (21). The analogous target used in this task for fungi is the internal transcribed spacer (ITS) region. Numerous tools and databases have been developed to assist with the automated analysis of sequence data to determine the taxonomic affiliation of each sequence analyzed.

It is important to note that the end product is a standardized approach rather than an optimized one: If the method recommended or available in academic or other U.S. national agencies is correctly applied, the user will generate a genetic inventory that is free from experimental anomalies and is (to the best of current knowledge) representative of the DNA types that are actually present on the surfaces sampled. To clarify, it is possible that variants of the methodology can recover higher yields or reduce known biases, but the NASA PP Program did not have the resources to investigate every experimental combination in what is a rapidly evolving field of technology. These experimental steps include, but are not limited to, sample collection, sample processing, and sample analysis.

During 2000-2010, scientists employed a number of different sample analysis methodologies, both as tools to analyze relative performance of other elements of the end-to-end molecular analyses process and as generators of diversity data about the environments sampled $(13,21)$. It quickly became apparent that traditional $16 \mathrm{~S}$ rDNA cloning and Sanger-sequencing approaches were not capable of elucidating an all-inclusive genetic inventory from low-biomass environments (22). These methods are limited by molecular biases that favor the amplification and/or amplicon ligation of abundant bacterial taxa and hence mask the presence of less abundant taxa. DNA microarray analyses were found to be superior to conventional 16S rRNA gene cloning and Sanger-sequencing strategies in all aspects of microbial diversity analysis, with one limitation: The methodology is based on a detection of hybridization to an array of oligonucleotides from previously described/sequenced organisms (22). It is therefore unreliable for detecting and identifying previously unreported taxa, particularly at the OTU level. During the 2010s, the most comprehensive inventory was obtained using Titanium 454 FLX tag-encoded pyrosequencing (8). This high-throughput DNA sequencing methodology is capable of detecting the presence of thousands of different microbial DNA sequences (previously reported and novel OTUs) from a single sample. However, key to the integrity of this approach is the need to analyze an appropriate target sequence: Long-read ( 350 base pairs) technology yielded good data for genetic inventory purposes, whereas a shorter tag sequence (<80 base pairs) was shown to be susceptible to interference from spurious non-sample DNA and provided insufficient resolution ability in the downstream data analysis. During this time period, pairedend Illumina-based next generation sequencing (NGS) technology emerged, but the approach still targeted amplicons (23).

Subsequently in 2015 , metagenomics was identified as a maturing technology that could potentially be used to detect the widest possible spectrum of microbes, including those of PP interest $(14,24)$. Microbes such as radiation-resistant psychrophiles, anaerobes, etc. are likely to withstand spaceflight conditions (launch to landing, particularly if protected within spaceflight hardware) and may be able to survive on an extraterrestrial planet (forward contamination). High-throughput metagenomics sequencing and bioinformatics pipelines have the potential to rapidly identify the majority of the microbial burden and to 
classify those microbes into PP-relevant categories. The Illumina-based 150-bp approach is still patchy since long reads are needed to understand the functionality of the microbes. The PacBio Seq did not serve the purpose for environmental metagenome sequencing since this technology was designed to get longer reads from pure DNA. The recent advent of Oxford Nanopore Technology (ONT), MinION, revolutionized the industry due to the miniaturization of the hardware, its portability, and its capability to sequence longer reads ( $>3$ to $5-\mathrm{kb}$ ) (25). The generation of longer reads will benefit our ability to understand the functional properties of the target microorganisms, but the ONT MinION method requires higher input of the biomolecules, unlike the short-read ( 150 bp) Illumina-based method. Based on these studies, it is recommended that a hybrid technology that combines both Illumina and ONT MinION methods be explored since it would better serve the NASA PP Program.

\subsubsection{Increased Understanding of Spacecraft-Associated Organisms and Extreme, Relevant Environments}

The use of modern molecular analytical methods to rapidly detect, classify, and examine the presence of PP-relevant organisms and genes will narrow down microbes that can survive spaceflight. This will serve as a model for microbial survival on Mars and Europa or any other terrestrial planet research. Datamining all the metagenomics and NGS data that have been generated so far (from spacecraft assembly facilities [SAF], ISS, and spacecraft) to identify the complete spectrum of organisms is needed so that these metagenomic data can be functionally analyzed to determine genes that support life or support the survival of PP-relevant microorganisms in extreme space conditions.

\subsubsection{Computational Bioinformatic Pipeline and Database Development}

It is now established that shotgun metagenomics sequencing has the ability to detect, quantify, and assess potential metabolisms of both cultivable and uncultivable microorganisms (14, 24). However, there are numerous software packages and sequence databases available for understanding the functionality of the microorganisms detected, and it is unknown how well (or poorly) various combinations of software packages and sequence databases perform at classifying metagenomics sequences. There is a big gap in the metagenome sequence bioinformatics analysis, and future studies are recommended to test the ability of modern metagenomics pipelines to help NASA define new PP requirements for detecting and understanding the functionality of microorganisms on space hardware for future missions.

The amount of data produced from various sequencing methods used for the NGS analysis is far beyond what can be analyzed manually. Accordingly, the molecular biology community has developed a series of tools and approaches to process the raw rRNA gene sequence data and interpret microbial community structure with statistical confidence; novel tools and strategies are also continually emerging. The key steps taken in the data analysis of sample-associated microbial diversity are (1) feeding the raw sequence data into appropriate bioinformatic and biostatistical pipelines to derive taxonomic affiliation, microbial diversity, community characteristics, and richness; (2) comparing the community structure and shared membership between samples; and (3) determining the occurrence of various microbial lineages to generate hypotheses about those that are of greatest concern to Planetary Protection.

The phylogenetic affiliations and taxonomy calling for the Sanger-sequencing approach is straightforward and well-established. However, while DNA microarray, Illumina, and MinION technologies share a similar goal (the thorough elucidation of biodiversity present in a given sample), each method makes use of its own intrinsic taxonomy calling system for assigning taxonomy to specific sequences. Since these technologies are based on different approaches (probe hybridization to $1.5 \mathrm{~kb}$ for PhyloChip vs. sequencing of $<150$ base pairs for Illumina vs. $>3$ kilo bases for MinION), attempting to directly compare biodiversity profiles resulting from NGS is challenging. Beyond the sequence analysis and taxonomy 
assignment, a suite of publicly available and in-house bioinformatic and biostatistical tools need to be developed to interpret microbial community structure. Since no single molecular technique or statistical sequence analysis tool can answer all of the questions pertaining to microbial ecology that can be applied to various approaches and tools for analyzing and interpreting the sequence data.

The end-to-end capability (sample collection to analysis) for low-biomass samples need to be tested repeatedly for its reproducibility on relevant spacecraft environment samples of opportunity. In conjunction with the bioinformatics approaches, these NGS studies will provide the newest data on the occurrence of rDNA sequences (as a surrogate for presence of the organism) on spacecraft and associated surfaces. These modern NGS molecular techniques should also be able to achieve $99 \%$ coverage (of the detected number of sequences compared to the predicted total diversity) for a spacecraft sample set.

Future NGS technology should be able to (1) systematically collect, process, and archive nucleic acids from spacecraft-related environments, and (2) effectively assess, using currently available analysis technologies, the diversity of microorganisms potentially present on spacecraft and associated surfaces. These capabilities would enable future NGS methods to generate the most comprehensive (bacterial, archaeal, and fungal) assessment of SAF-associated biodiversity to date. Lessons learned, conclusions, and recommendations will provide a modernized standard approach to implementing a comprehensive microbial diversity for missions today, facilitating a risk-based analysis analogous to the venerable NSA for the analysis of mesophilic, heterotrophic, spore-forming organisms.

\section{Discussion and Conclusions}

The NSA has allowed NASA to mitigate biological outbound spacecraft contamination and verify compliance with PP requirements for robotic spacecraft to Mars and Europa. Mars 2020 and Europa Clipper have experienced implementation challenges with the NSA being the only approved approach and have had to rely on mission-sponsored technology development to meet their respective mission requirements for genetic inventory and probabilistic risk assessment. This reactive engineering response resulted in each project mitigating a moderate to high project level risk to meet PP requirements and required multiple years of development that culminated around the project's critical design review timeframe. Given this inflection point, more complex missions would experience a greater impact from being limited to using a culture-based approach, resulting in the utilization of a greater amount of project resources (e.g., schedule most notable) to address this knowledge gap. Hence, this put projects at risk as they will be required to develop an end-to-end approach to be responsive to biological contamination requirements.

It is a high priority in the upcoming decade to mature a nucleic acid-based approach, adapting it from industry and academia approaches, to develop a standard spacecraft methodology for biological contamination assessments for low-biomass spacecraft surfaces in the following areas:

- $\quad$ sampling and sample processing (swab to sequencing) capability from-low biomass spacecraft surfaces,

- viable organism enumeration,

- phylogenetic identification assessment of the broadest spectrum of organisms on the surface,

- generation of a high-resolution characterization of microbial traits and biochemical capabilities of biological contamination control relevance,

- continued development of understanding of microbes in extreme environments and tolerance to sterilization modalities,

- bioinformatic pipeline and database standardization,

- feedback loop for use of nucleic acid data into supplementary culture-based studies,

- rapid ability to assess PP-relevant organisms, and 
- development of a NASA / science community-approved integrated verification approach to include demonstration with side-by-side NSA comparison for evaluation to understand the interdependencies for supplementing modern assays.

This technology development will allow for data rich evaluations of biological contamination that will assist policy makers, engineers, and scientist to manage the science risk and PP risk for both outbound spacecraft, during operational human Mars mission scenarios, and for inbound sample-return decision making.

\section{Summary}

The 1960s spore culture-based approach has allowed NASA to assess biological contamination on current spacecraft, but the approach significantly limits the biological contamination knowledge that is required for missions with increasingly sensitive life detection instruments, human to Mars, assessing icy worlds probabilistic risk requirements, and sample return. Modernizing this toolkit with capability-driven research to improve methods to both understand and minimize the biological contamination for outbound spacecraft, operational monitoring for humans, and for returned samples would ensure that PP has methodologies within the next decade that are mission-enabled to support both science contamination control and PP requirements.

\section{References}

1. J. N. Benardini, 3rd, M. T. La Duc, D. Ballou, R. Koukol, Astrobiology 14, 33 (Jan, 2014).

2. J. R. Puleo et al., Appl. Environ. Microbiol. 33, 379 (Feb, 1977).

3. R. Hendrickson, G. Kazarians, J. N. Benardini, 3rd, Astrobiology, (Jun 18, 2020).

4. R. Hendrickson et al., Astrobiology, (Sep 9, 2020).

5. G. Horneck, D. M. Klaus, R. L. Mancinelli, Microbiol. Mol. Biol. Rev. 74, 121 (Mar, 2010).

6. D. A. Newcombe et al., Appl. Environ. Microbiol. 71, 8147 (Dec, 2005).

7. W. W. Schubert, R. A. Beaudet, Astrobiology 11, 213 (Apr, 2011).

8. M. T. La Duc, P. Vaishampayan, H. R. Nilsson, T. Torok, K. Venkateswaran, Appl. Environ. Microbiol. 78, 5912 (Aug, 2012).

9. NASA. (National Aeronautics and Space Administration, Washington, D.C., 2011).

10. M. T. La Duc, W. Nicholson, R. Kern, K. Venkateswaran, Environ. Microbiol. 5, 977 (Oct, 2003).

11. M. T. La Duc, K. Venkateswaran, C. A. Conley, Astrobiology 14, 15 (Jan, 2014).

12. P. Vaishampayan, S. Osman, G. Andersen, K. Venkateswaran, Astrobiology 10, 499 (2010).

13. K. Venkateswaran, M. T. La Duc, P. Vaishampayan. (Jet Propulsion Laboratory, California Institute of Technology, Pasadena, CA., 2012), vol. 1 and 2, pp. 1-117.

14. T. Weinmaier et al., Microbiome 3, (2015).

15. M. H. Andersen, S. J. Mcllroy, M. Nierychlo, P. H. Nielsen, M. Albertsen, Syst. Appl. Microbiol. 42, 77 (Jan, 2019).

16. M. J. Leon et al., Appl. Environ. Microbiol. 80, 3850 (Jul, 2014).

17. J. Goris et al., Int. J. Syst. Evol. Microbiol. 57, 81 (2007).

18. N. K. Singh, J. M. Wood, S. S. Mhatre, K. Venkateswaran, Appl. Microbiol. Biotechnol. 103, 4483 (Jun, 2019).

19. P. Vaishampayan et al., ISME J 7, 312 (02//print, 2013).

20. H. Pan et al., Food Control 112, 107140 (2020/06/01/, 2020).

21. N. R. Pace, Science 276, 734 (May 2, 1997).

22. M. T. La Duc et al., Appl. Environ. Microbiol. 75, 6559 (October 15, 2009, 2009).

23. J. G. Caporaso et al., Proceedings of the National Academy of Sciences 108, 4516 (2011).

24. N. K. Singh, J. M. Wood, F. Karouia, K. Venkateswaran, Microbiome 6, 214 (Dec 4, 2018).

25. S. L. Castro-Wallace et al., Sci. Rep. 7, 18022 (2017/12/21, 2017). 